\title{
Is Acute Myeloblastic Leukemia in Children Under 2 Years of Age a Specific Entity? A Report from the FRENCH ELAM02 Study Group
}

\author{
S. Blais ${ }^{1,2}$, H. Boutroux ${ }^{2,3}$, M. Pasquet ${ }^{4}$, T. Leblanc ${ }^{5}$, O. Fenneteau ${ }^{6}$, V. Gandemer ${ }^{7}$, Y. Bertrand ${ }^{8}$, \\ S. Ducassou' ${ }^{9}$, G. Michel ${ }^{10}$, B. Nelken ${ }^{11}$, A. Petit ${ }^{2,3}$, W. Cuccuini ${ }^{12}$, E. Gouache ${ }^{2,3}$, Marceau-A. Renaut ${ }^{13}$, \\ A. Baruchel ${ }^{5}, \mathrm{H}$. Lapillonne ${ }^{14}, \mathrm{G}$. Leverger 2,3
}

Correspondence: Sophie Blais (e-mail: sblais@chi-poissy-st-germain.fr).

\begin{abstract}
The clinical and biological characteristics of children under 2 years (infants) with acute myeloid leukemia (AML) are different from those of older children. We aimed to describe the specific characteristics of this population and the potential factors that influence the prognosis. We analyzed data concerning 438 children with newly-diagnosed AML treated in the ELAM02 protocol between March 2005 and December 2011, of which 103 were under 2 years old at diagnosis. The evaluation criteria were overall survival (OS) and event-free survival (EFS) of infants vs older children. The clinical and biological features were secondary criteria. Infants presented more frequent extra-medullary presentation than older children. They had a significantly higher proportion of skin lesions and central nervous system involvement (15\% vs 3\%, $\mathrm{p}<0.0001$ and $26 \%$ vs $12 \%, \mathrm{p}=0.0005$, respectively). The global incidence of KMT2A rearrangements was nearly $55 \%$ for infants vs $11 \%$ for older children ( $p<0.0001$ ). Median 5 -year OS was $70.4 \%$ for infants vs $71.4 \%$ for older children $(p=0.83)$. Five-year EFS was $67 \%$ for infants vs $58 \%$ for older children $(p=0.27)$. Infants with AML represent a cohort of patients with specific clinical and biological features. These remarkable differences had no significant impact on their outcome in the ELAMO2 protocol.
\end{abstract}

\footnotetext{
The authors have no conflict of interest to disclose for this work.

${ }^{1}$ Pediatric Department, Poissy Hospital, Poissy, France

${ }^{2}$ Department of Pediatric Hematology, Trousseau Hospital (AP-HP), Paris, France ${ }^{3}$ Sorbonne Université, Inserm, Centre de Recherche Saint-Antoine, CRSA, F75012, Paris, France

${ }^{4}$ Department of Pediatric Hematology, Hôpital des Enfants, Toulouse, France ${ }^{5}$ Pediatric Hematology Department, Robert Debré Hospital, Paris, France

${ }^{6}$ Biological Hematology Department, Robert Debré Hospital, Paris, France ${ }^{7}$ Department of Pediatric Hematology and Oncology, University Hospital of Rennes, Rennes, France

${ }^{8}$ Department of Pediatric Hematology and Oncology, University Hospital of Lyon, Lyon, France

${ }^{9}$ Department of Pediatric Hematology and Oncology, University Hospital of Bordeaux, Bordeaux, France

${ }^{10}$ Department of Pediatric Hematology and Oncology, Timone Children Hospital and Research Unit, Aix-Marseille University, Marseille, France

${ }^{11}$ Pediatric Hematology Department, University Hospital of Lille, Lille, France

${ }^{12}$ Hematology Laboratory, Saint-Louis Hospital, AP-HP, Paris, France

${ }^{13} \mathrm{CHU}$ Lille, Laboratory of Hematology, Lille, France

${ }^{14}$ Laboratory of Hematology, Trousseau Hospital (AP-HP) Paris, France

Copyright () 2019 the Author(s). Published by Wolters Kluwer Health, Inc. on behalf of the European Hematology Association. This is an open access article distributed under the terms of the Creative Commons Attribution-Non

Commercial-No Derivatives License 4.0 (CCBY-NC-ND), where it is permissible to download and share the work provided it is properly cited. The work cannot be changed in any way or used commercially without permission from the journal.

HemaSphere (2019) 3:6(e316)

Received: 12 June 2019 / Received in final form: 16 October 2019 / Accepted: 19 October 2019

Citation: Blais S, Boutroux H, Pasquet M, Leblanc T, Fenneteau O,

Gandemer V, Bertrand Y, Ducassou S, Michel G, Nelken B, Petit A, Cuccuini

W, Gouache E, Renaut MA, Baruchel A, Lapillonne H, Leverger G. Is Acute

Myeloblastic Leukemia in Children Under 2 Years of Age a Specific Entity? A

Report from the FRENCH ELAM02 Study Group. HemaSphere, 2019;3:6.

http://dx.doi.org/10.1097/HS9.0000000000000316
}

\section{Introduction}

Acute myeloblastic leukemia (AML) is a rare disease in pediatrics, accounting for approximately 75 to 80 cases per year in France. ${ }^{1}$ The frequency peaks in young children under 2 years of age (infants) and the incidence then increases until adulthood. ${ }^{1}$ Despite recent progress in the understanding and management of childhood AML, there is still a high risk of relapse $(40 \%$ to $50 \%)$. Nevertheless, recent standards of care enable overall survival (OS) of $60 \%$ to $70 \%$ in most developed countries. ${ }^{2}$ This improvement has been achieved due to new treatment strategies, better supportive care, and more effective patient stratification. ${ }^{3,4} \mathrm{High}$-risk patients require more intensive treatment, which can include allogeneic hematopoietic stem cell transplantation. ${ }^{5}$

Infants with AML appear to constitute a specific subgroup in terms of both clinical presentation and biological characteristics. ${ }^{6,7}$ In addition, because of their young age, this subgroup may have an underlying predisposition. Indeed, recent data in the field of clonal architecture of AML suggest different clonal evolution in this population than in the model described for adult patients. ${ }^{8}$ Thus, an age at diagnosis of under 2 years may indicate a different entity. We used the term of infants for these children as Masetti et al. ${ }^{7}$

We aimed to study the clinical and biological presentation at the time of diagnosis of children under 2 years of age with AML. We compared cytogenetic and molecular markers and treatment outcome of infants vs that of children over 2 years of age in the ELAM02 trial. ${ }^{9}$ 


\section{Materials and methods}

\section{Study population}

This retrospective multi-center analysis included all patients with AML treated in the ELAM02 protocol from March 2005 to December 2011 in 28 medical centers in France.

Inclusion criteria were patients aged between 0 and 18 years at diagnosis; an AML not previously treated; French-AmericanBritish (FAB) subtype M0, M1, M2, M4, M4 eosinophil, M5, M6, or M7, unclassifiable or myelodysplasia (blastosis $>20 \%$ ), or isolated chloroma. The written consent of both parents and the child (depending on age) to participate in the study was received for each case.

Children with Down syndrome, AML FAB subtype M3 or variant M3 and secondary AML, occurring after the treatment of a first malignancy or during the course of a predisposing genetic disease, were excluded.

CNS (central nervous system) involvement was defined by cerebrospinal fluid with 5 white bloods cells $/ \mathrm{mm}^{3}$ or more, or less than 5 white bloods cells $/ \mathrm{mm}^{3}$ with blasts on the smear after cytocentrifugation, and/or clinical symptoms. Complete remission (CR) was defined by a normal cell density with less than $5 \%$ blasts by myelogram with a normal blood count and the disappearance of clinical symptoms. The presence of skin lesions was verified in the medical records.

\section{Biological analyses}

A centralized hematological review of blood and bone marrow samples was performed at diagnosis and after hematological recovery following the first course of consolidation.

A cytogenetics analysis was performed at diagnosis for all patients, including: standard karyotype (with the analysis of at least 20 mitoses) and FISH analysis, searching for KMT2A rearrangement and inversion in chromosome 16. A central review was conducted by N. Dastugue (Laboratory of Hematology Purpan, Toulouse). Karyotypes were classified as follows CBFrearranged, KMT2A-rearranged and other aberrations.

Prospective national biobanking of all blood and bone-marrow samples at diagnosis and during the treatment of the patients involved in the ELAM02 trial was performed (Laboratory of Hematology - Trousseau hospital, Paris).

Molecular biology analysis at diagnosis and during treatment was performed in Lille and Paris (Laboratory of Hematology Pr. Lapillonne and Pr. Preudhomme). The analysis at diagnosis included a search for gene mutations by PCR (polymerase chain reaction). Specific fusion transcripts (AML1-ETO, CBFBMYH11, KMT2A transcripts, NUP98 transcripts, ETO2GLIS2) were analyzed according to the cytogenetics results. Genomic DNA from bone-marrow aspirates at diagnosis was also studied by high-throughput sequencing (HTS) of 36 genes recurrently mutated in AML. The results of these analyses were recently published. ${ }^{10}$

\section{Treatment}

Treatment is summarized in Figure 1. The induction regimen combined mitoxantrone and cytarabine, with an evaluation of chemosensitivity by a myelogram at D15, possibly repeated at D20. The first consolidation treatment combined high-dose cytarabine and amsacrine, starting on either D21 for chemoresistant forms or D35 for chemo-sensitive forms. CR was estimated at the end of this stage of treatment. Patients received two consolidation treatments except for those with an identical HLA donor and an indication for a matched allograft. CNS prophylaxis or treatment was included, consisting of triple intrathecal injections. Chemotherapy doses were reduced to 2 thirds the normal dose for children under 6 months of age and three quarters the normal dose for children aged from 6 months to 1 year or those weighing less than $10 \mathrm{~kg}$.

Patients were stratified into 3 groups for allogeneic bonemarrow transplantation. For group 1 "standard risk" including $\mathrm{t}$ $(8 ; 21)(\mathrm{q} 22 ; \mathrm{q} 22)$ or inv $(16)(\mathrm{p} 13 \mathrm{q} 22) / \mathrm{t}(16 ; 16)(\mathrm{p} 13 ; \mathrm{q} 22)$, allogeneic bone-marrow transplantation was not recommended, even if an HLA matched family donor was available. Group 2 "intermediate risk" included patients who did not fit into either group 1 "standard risk" or group 3 "high risk". The group 3 "high risk" included patients with secondary leukemia, absence of cytologic remission after consolidation 1 or patients with adverse cytogenetic or molecular abnormalities (monosomy 7 , abn5q, t(6;9)(p23;q34), t(10;11)(p11-14;q23), t(6;11)(q27;q23), $\operatorname{inv}(3)(\mathrm{q} 21 \mathrm{q} 26)$ or $\mathrm{t}(3 ; 3)(\mathrm{q} 21 ; \mathrm{q} 26)$, complex karyotype, presence of FLT3-ITD and ratio mutated allele/wild-type allele $>0,4)$. For group 3, allogeneic bone-marrow transplantation was recommended following the first CR with an HLA matched family donor or an unrelated matched donor and/or umbilical cord blood.

The ELAM02 protocol included a therapeutic randomized research protocol. Half of all patients with persistent $C R$ received interleukin-2 as maintenance treatment after chemotherapy.

\section{Follow up assessment}

Patients were regularly followed up to five years after CR. The following data were retrieved from the ELAM02 protocol database: clinical and biological presentation at the time of diagnosis, cytogenetic and molecular markers, and treatment outcome.

\section{Statistical methods}

Outcome was defined by overall survival (OS) and event-free survival (EFS). OS was calculated from diagnosis to death from any cause. Events were defined for EFS as refractory disease, relapse after CR, or death from any cause. Survival curves were calculated using the Kaplan-Meier method. ${ }^{11}$ Statistical significance of age at diagnosis was tested using the log-rank test. ${ }^{12}$

\section{Results}

\section{Patient characteristics}

A total of 438 patients with AML were included in this protocol. Their initial clinical and biological features are presented in Table 1. Among 438 patients, 103 were less than 2 years old at diagnosis, representing almost a quarter of patients. Among these 103 children, 54 were less than 1 year old at diagnosis. In this population, the proportion of boys tended to be higher than in older children (60 of 103 vs 179 of 335, p=0.42). The median follow-up of infants was 4.75 years.

\section{Clinical characteristics (Table 1)}

The initial clinical characteristics of infants with AML were distinct from those of the other children. Infants had more frequent extra-medullary symptoms of AML than older children. 


\section{INDUCTION}

myelogram at D15-possibly D20

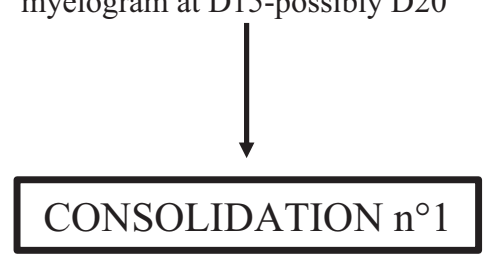

started at D21 or as of aplasia output between D35-D42

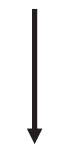

myelogram at D28 after consolidation $n^{\circ} 1$
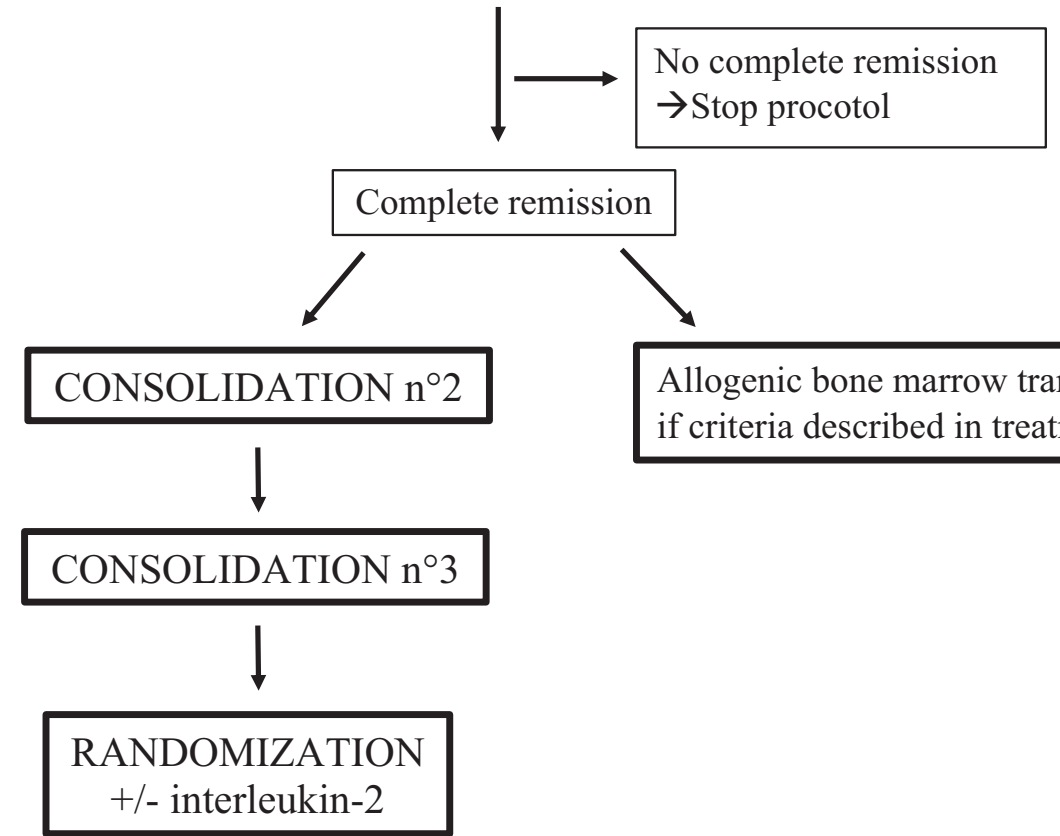

Figure 1. ELAMO2's treatment plan.

Indeed, they had a significantly higher proportion of skin lesions and CNS involvement $(15 \%$ vs $3 \%, \mathrm{p}<0.0001$, and $26 \%$ vs $12 \%, \mathrm{p}=0.0005$, respectively).

\section{Biological characteristics (Table 1)}

The biological characteristics of infants with AML were distinct from those of older children. There was a higher incidence of acute monoblastic and megakaryoblastic leukemia, M5 and M7 subtypes in the FAB classification. This difference was significant for the M7 subgroup ( $17 \%$ vs $3 \%, p<0.0001)$. In contrast, the proportion of M2 and M4 leukemia, including core binding factor $(\mathrm{CBF})$ leukemia, was lower in the infant group: $3 \%$ vs $29 \%$ and $12 \%$ vs $21 \%$, respectively.

The percentage of hyperleukocytosis (leukocytosis $\geq 50.000 /$ $\mathrm{mm}^{3}$ ) was essentially the same for both groups, approximately $30 \%$. There was a non-significant difference between 0 to 1 year $(19 / 55)$ vs 1 to 2 years of age (11/48).

The cytogenetic abnormalities of AML in infants were different from those found in older children (Fig. 2A). The global incidence of KMT2A rearrangements was nearly $55 \%$ for infants vs $11 \%$ for older children $(\mathrm{p}<0.0001)$. In contrast, the incidence of $\mathrm{CBF}$ abnormalities was higher for older children, nearly $29 \%$ vs $5 \%$ for infants $(\mathrm{p}<0.0001)$. The distribution within cytogenetic groups was therefore different, $44 \%$ in group 3 "high risk" for infants, approximately $50 \%$ in group 2 "intermediate risk" for all patients, and $27 \%$ in group 1 "standard risk" for older children. Moreover, the proportion of normal karyotypes tended to be lower in infants (9\% vs $31 \%$ ). The principal molecular aberrations of AML were also different according to age (Fig. 2B). Overall, infants had fewer molecular abnormalities. The average number of mutations tended to be lower in infants (0.53 vs 1.59 ). The prevalence of the FLT3 mutation increased with age, almost $6 \%$ (1 patient with FLT3-ITD and 5 patients with FLT3-TKD) for infants vs almost $27 \%$ for older children. The prevalence of RAS mutations was similar in the two groups ( $37 \%$ for older children vs $30 \%$ for infants). Reported mutations in GATA2 are somatic mutations.

In the group of less than one year and the group between 1 and 2 years of age the incidence of AML subtypes by the FAB 
ELAM02's Results.

\begin{tabular}{|c|c|c|c|c|c|}
\hline & \multicolumn{2}{|c|}{$<2$ years old } & \multicolumn{2}{|c|}{$>$ 2years old } & \multirow[b]{2}{*}{$p$ value } \\
\hline & Number & $\%$ & Number & $\%$ & \\
\hline Patients & 103 & $24 \%$ & 333 & $76 \%$ & \\
\hline \multicolumn{6}{|l|}{ Sex } \\
\hline Girls & 43 & $42 \%$ & 154 & $46 \%$ & \\
\hline \multicolumn{6}{|l|}{ Clinical characteristics } \\
\hline Chloromas & 8 & $8 \%$ & 13 & $4 \%$ & 0.1095 \\
\hline No skin involvement & 88 & $85 \%$ & 324 & $97 \%$ & \\
\hline Central nervous system + & 27 & $26 \%$ & 40 & $12 \%$ & 0.0005 \\
\hline Central nervous system - & 76 & $74 \%$ & 293 & $88 \%$ & \\
\hline \multicolumn{6}{|l|}{ Biological characteristics } \\
\hline FAB type & & & & & \\
\hline MO & 2 & $2 \%$ & 26 & $8 \%$ & \\
\hline M6 & 3 & $3 \%$ & 9 & $3 \%$ & \\
\hline M7 & 18 & $17 \%$ & 9 & $3 \%$ & $<0.0001$ \\
\hline Other & 9 & $9 \%$ & 8 & $2 \%$ & \\
\hline Hyperleukocytosis & 30 & $29 \%$ & 97 & $29 \%$ & \\
\hline No hyperleukocytosis & 73 & $71 \%$ & 236 & $71 \%$ & \\
\hline \multicolumn{6}{|l|}{ Cytogenetic risk } \\
\hline group 1 & 4 & $4 \%$ & 91 & $27 \%$ & \\
\hline group 2 & 52 & $50 \%$ & 182 & $55 \%$ & \\
\hline group 3 & 42 & $44 \%$ & 59 & $18 \%$ & \\
\hline failure & 2 & $2 \%$ & 1 & $0 \%$ & \\
\hline \multicolumn{6}{|l|}{ Outcome } \\
\hline Complete remission & 89 & $86 \%$ & 300 & $90 \%$ & 0.284 \\
\hline
\end{tabular}

classification was similar. The incidence of the M7 subtype was $17 \%$ vs $19 \%$, respectively. There was no significant difference in the incidence of cytogenetic abnormalities of AML between the 2 age groups. The incidence of KMT2A rearrangements was $54 \%$ in the group of less than one year vs $55 \%$ for the group between 1 and 2 years of age.

\section{Treatment outcome}

There were no significant differences in the CR or induction failure rates or incidence of relapse between infants and children older than 2 years of age. Indeed, $86 \%$ of children younger than 2 years of age were in remission at the end of consolidation $1 v \mathrm{~s}$ $90 \%$ of the children older than 2 years of age $(p=0.28)$. The incidence of relapse was the same for both groups (31\% vs 39\%), most (almost $70 \%$ ) relapses were medullary.

We found no prognostic markers of relapse in either group. There were no significant differences in OS or EFS between patients under 2 years of age and older (Fig. 3). Median 5-year OS was $70.4 \%$ for children under 2 years of age vs $71.4 \%$ for older children $(p=0.83)$. Five-years EFS was $67 \%$ for the group younger than 2 years of age and $58 \%$ for the older group $(\mathrm{p}=$ 0.27 ). The children generally died from progression of leukemia; there was no difference between the 2 groups for the cause of death $(\mathrm{p}=0.93)$.

There was also no impact of KMT2A status on OS and EFS. Median five-year OS was $72.9 \%$ for children with KMT2A rearrangements vs $73 \%$ for those without $(\mathrm{p}=0.99)$ from the whole ELAM02 study. Median 5-year EFS was $68.2 \%$ vs $66.5 \%$ $(\mathrm{p}=0.65)$, respectively.

During CR after the first course of consolidation (CR1), 19\% of infants had hematopoietic stem cell transplantation vs $29 \%$ of the older children. A total of 273 children were treated with chemotherapy without bone marrow transplant: $67 \%$ of infants vs $61 \%$ of older children. Data concerning hematopoietic stem cell transplantation was missing for $10 \%$ of the children in each group.

Among infants, we found a significant poor impact of M7 subtype on prognosis, both for EFS and OS. Indeed, infants with M7 AML had a 5-years EFS of 53\%, vs 77\% for infants with non M7 AML $(p=0.03)$. And 5-years OS was respectively $47 \%$ and $70 \%$ for infants with M7 AML and non M7 AML (p=0.02). 


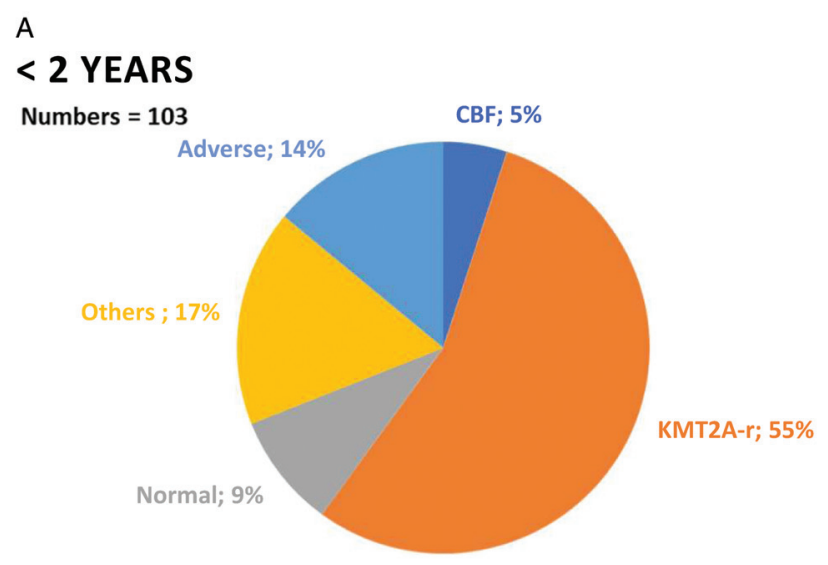

B

\section{$>2$ YEARS \\ Numbers $=335$}

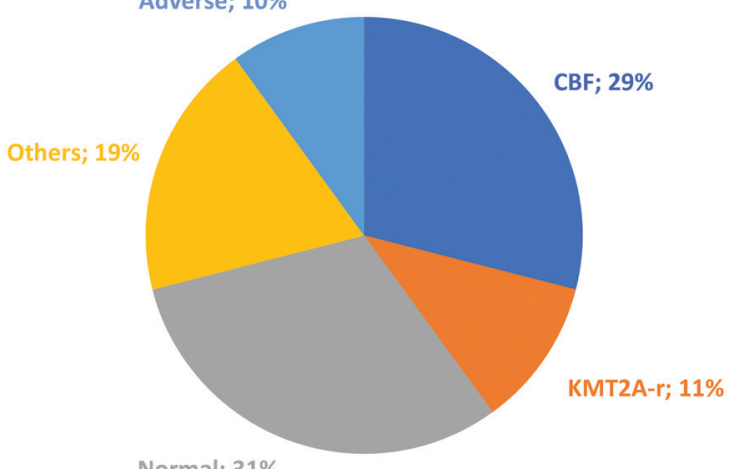

$<2$ YEARS

Numbers $=103$

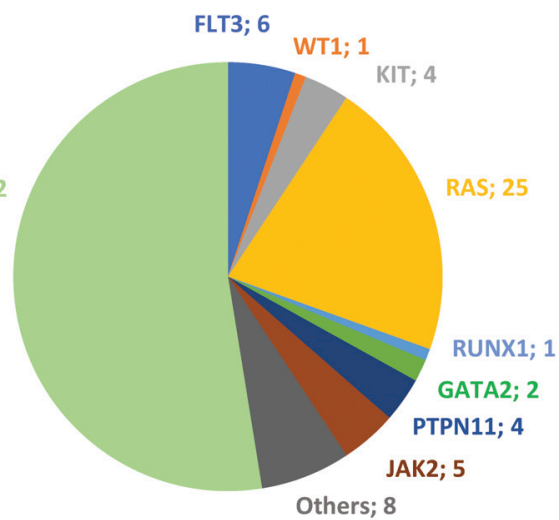

$>2$ YEARS

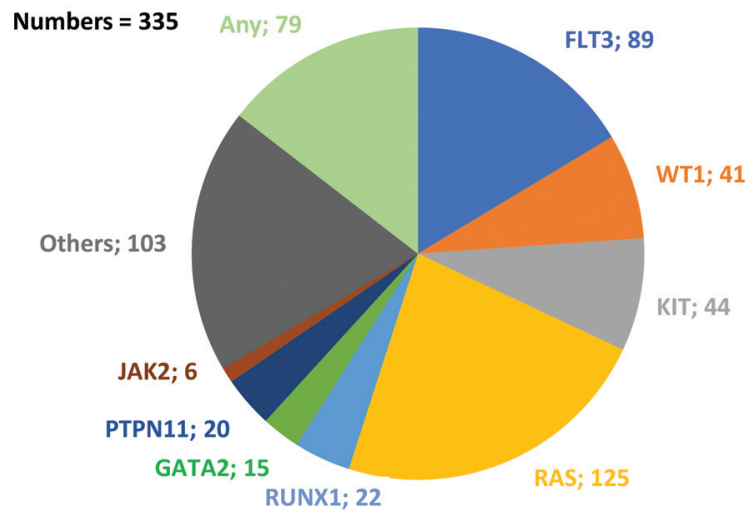

Figure 2. Distribution of the cytogenetic in \% (A) and molecular (B) abnormalities reported in children with AML younger and older than 2 years of age in ELAM02.
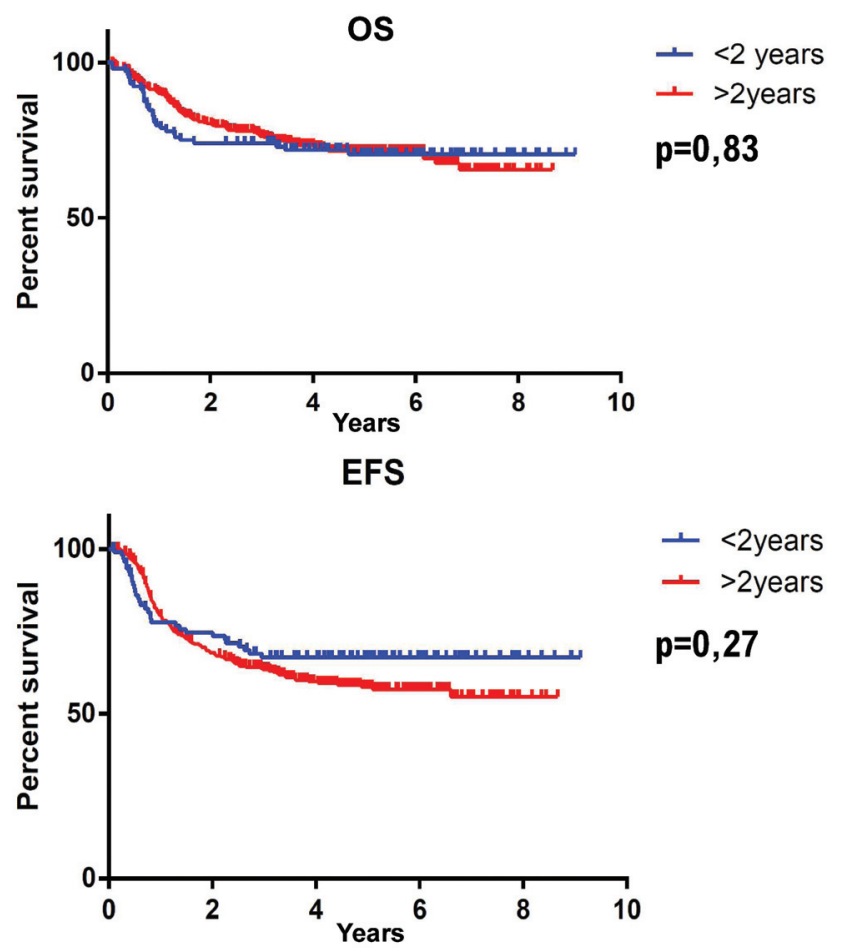

Figure 3. Overall survival (OS) and Event free survival (EFS) in ELAMO2 for children younger and older than 2 years of age.

\section{Discussion}

This study, based on the ELAM02 protocol, confirms that infant AML is a distinct entity with a specific clinical and biological presentation, implying potentially different oncogenic mechanisms. However, these specificities do not appear to affect the global prognosis of infants, either in terms of EFS or OS, consistent with the data in the literature. ${ }^{7}$

Independently of prognosis, there has long been an interest in this age category of children, as shown in the study in 1991 at the Saint-Louis Hospital (Paris). ${ }^{6}$

Children under 2 years old (infants) with AML represent a quarter of all children with AML treated in France, hence the necessity of studying this sub-population. Within infants, the age distribution is homogeneous. The subtypes, by FAB classification, and incidence of cytogenetic abnormalities were similar.

The results of the ELAM02 protocol showed a higher incidence of extramedullary involvement in children under 2 years of age. In infants, skin involvement may be a prognostic factor in childhood AML. ${ }^{13}$ The higher incidence of CNS disease in infants has been found in several other studies in France and other countries. ${ }^{7,14-17}$ This difference may be due to the immaturity of the blood-brain barrier in infants, which does not fully develop until the age of six months. ${ }^{18}$ It may also be due to the greater vasculature of the leptomeninges of infants and preschool children. ${ }^{19}$ Several studies have also documented a higher incidence of other extramedullary organ involvement ${ }^{15}$ without providing a hypothesis to explain this difference. CNS involvement at diagnosis is associated with a higher incidence of CNS 
relapse, but it does not appear to influence OS of children with AML. ${ }^{20}$ The ability to migrate into other organs in infants may also be due to intrinsic characteristics of the blast cells themselves, due to their immaturity and/or cytological subtype. Indeed, in our experience, the prevalence of skin involvement is also more frequent in infants. Gouache et al reported in 2018 a poorer pronostic in patient with leukemia cutis. ${ }^{13}$ This data may be crucial for earlier and more accurate diagnosis in infants.

The data for FAB subtypes showed a higher incidence of acute monoblastic and megakaryoblastic leukemia, M5 and M7 in the FAB classification, which is consistent with previously published studies. ${ }^{3,14,15}$ We confirmed in the infants population that M7 subtype is related to a poorer outcome than other subtypes, as for EFS and OS. ${ }^{21}$ Another result in common with those reported in the literature was the low proportion of the M1/M2 type in infants $(6 \%)$ relative to older children $(48 \%)$, for whom they represent the most common subtype. However, our population of infants had a lower proportion of the M4 subtype than reported in other studies, of which some have shown the M4 and M5 subtypes to be good prognostic factors. ${ }^{22,23}$

The percentage of hyperleukocytosis in infants was similar to that in older children in the ELAM02 protocol. This result is different from those of other studies in other countries ${ }^{16,23,24}$ and those in which the age standard was set to one year. ${ }^{14}$

The chromosomal aberrations of AML in infants consisted largely of translocations involving chromosome band $11 \mathrm{q} 23 .^{25}$ The global incidence of $11 \mathrm{q} 23 /$ KMT2A rearrangements in our study was comparable with those of other studies, between $40 \%$ and $55 \% .^{7,14,15,24}$ Nevertheless, the very small number of additional cooperating oncogenic abnormalities suggests that the driving force of KMT2A translocations may be much greater. Studies are under way to address this issue, such as the study of clonal evolution of this subpopulation. New technologies, such as NGS (next generation sequencing), can also provide new information and better characterize the subpopulations. ${ }^{8}$ There are few other cytogenetic abnormalities that cooperate with KMT2A rearrangements, showing the predominant function of $K M T 2 A$ in the dysregulation of cell maturation and proliferation. Here, there was no difference in OS and EFS between $K M T 2 A^{+}$ and $K M T 2 A^{-}$patients.

The other distinctive trait of infant AML is the very low frequency of $\mathrm{CBF}$ rearrangements, never before one year of age, which is a favorable feature. ${ }^{7,26}$ A tendency towards a higher proportion of $12 \mathrm{p}$ abnormalities has been reported for infants in other studies ${ }^{15}$ and appears to characterize a cytogenetic group of patients with an adverse outcome. ${ }^{27}$ In the current international protocol MyeChild01, ${ }^{28}$ this cytogenetic group of patients will be classified as high risk and require more aggressive therapy.

The main molecular abnormalities of AML found in children younger than 2 years of age were few, with no impact on prognosis. The prevalence of mutations with a good prognostic profile appears to increase with age after 2 years, such as WT1. ${ }^{7}$ The AML predisposition syndrome GATA2 alteration was found in only $1 \%$ of the population of older children, probably suggesting a delay in the triggering of oncogenic alterations leading to AML.

The results of the ELAM02 protocol did not reveal any significant differences in OS or EFS between infants and older children. ${ }^{15,24}$ However, we found a trend towards dying earlier after diagnosis in the infant population, which can be explained by higher toxicity in infants. Indeed, the management of infants with AML did not differ from that of older children in ELAM02, except the adaptation of the doses of cytotoxic drugs. It is important that infants be treated in centers with recognized expertise.

There were no distinct clinical prognostic markers of relapse in either group. Future studies on biological abnormalities, in particular the subtype of AML, could focus on how these correlate with age and the risk of relapse.

A total of 273 of the 438 children were treated with chemotherapy without hematopoietic stem cell transplantation. There is no consensus of the indication of hematopoietic stem cell transplantation during CR1, in particular in infants. ${ }^{24}$ This will require specific studies on this subject.

\section{Conclusion}

In conclusion, infants with AML make up a subgroup of patients with their own clinical and biological features. They have more frequent extra-medullary symptoms of AML and had a significantly higher incidence of CNS involvement, $26 \%$ vs $12 \%, p=0.0005$. The cytogenetic abnormalities in infants are different from those found in older children; the incidence of KMT2A rearrangements is significantly higher in infants $(55 \%$ vs $12 \%$ ). These differences have no significant impact on their OS or EFS. OS of infants was more than $70 \%$ at 5 years in the ELAM02 protocol, comparable to the results obtained for older children. The primary impact of KMT2A rearrangements on cell proliferation and maturation requires further studies. These trials should define new cytogenetic and molecular features for the definition of subgroups of infants and children instead of only by age.

\section{Acknowledgments}

The authors wish to thank the following for their participation: Pr Guy Leverger, Armand Trousseau (Paris), Pr Simon Tabassome, Saint-Antoine (Paris), and Dr Anne Auvrignon, Armand Trousseau (Paris). We thank all participants in the ELAM02 protocol, as well as the medical centers that included children. We especially thank Dr Marlène Pasquet, Dr Thierry Leblanc, Pr Virginie Gandemer, Pr Yves Bertrand, Dr Stéphane Ducassou, Pr Gérard Michel, Dr Brigitte Nelken, Pr Arnaud Petit, and Pr André Baruchel. The ELAM02 protocol was a study under the aegis of the "Société Française de lutte contre les Cancers et les leucémies de l'Enfant et de l'adolescent (SFCE) » and the Belgian Society for Pediatric Hematology and Oncology (BSPHO). Pr Hélène Lapillonne, Armand Trousseau (Paris), centralized the molecular biology analysis at diagnosis and during treatment. Dr Odile Fenneteau, Robert Debré (Paris) reviewed the cytology data for all patients and Dr Wendy Cuccuini, Saint-Louis (Paris), Dr Nicole Dastugue, Purpan (Toulouse), and Pr Claude Preudhomme (Lille) reviewed the cytogenetic characteristics.

\section{References}

1. Goujon-Bellec S, Mollié A, Rudant J, et al. Time trends and seasonal variations in the diagnosis of childhood acute lymphoblastic leukaemia in France. Cancer Epidemiol. 2013;37:255-261.

2. Pui C-H, Carroll WL, Meshinchi S, et al. Biology, risk stratification and therapy of pediatric acute leukemias: an update. J Clin Oncol. 2011; 29:551-565.

3. Creutzig U, van den Heuvel-Eibrink MM, Gibson B, et al. Diagnosis and management of acute myeloid leukemia in children and adolescents: recommendations from an international expert panel. Blood. 2012; 120:3187-3205. 
4. Rasche M, Zimmermann M, Borschel L, et al. Successes and challenges in the treatment of pediatric acute myeloid leukemia: a retrospective analysis of the AML-BFM trials from 1987 to 2012. Leukemia. 2018;32:2167-2177.

5. Rubnitz JE. How I treat pediatric acute myeloid leukemia. Blood. 2012;119:5980-5988.

6. Gardembas-pain M, Flandrin G, Daniel M-T, et al. Acute myeloid leukemia in children less than two years old: clinical, cytologic and cytogenetic correlations comparison with other age categories. a clinical survey of 39 cases. Leuk Lymphoma. 1991;3:365-373.

7. Masetti R, Vendemini F, Zama D, et al. Acute myeloid leukemia in infants: biology and treatment. Front Pediatr. 2015;3:37.

8. Hirsch $P$, Zhang $Y$, Tang $R$, et al. Genetic hierarchy and temporal variegation in the clonal history of acute myeloid leukaemia. Nat Commun. 2016;7:12475.

9. https://clinicaltrials.gov/ct2/show/record/NCT00149162, Principal Investigator:Guy Leverger, M.D. Hopital Trousseau Paris, First Posted: September 8, 2005 Last Update Posted: February 12, 2009.

10. Marceau-Renaut A, Duployez N, Ducourneau B, et al. Molecular profiling defines distinct prognostic subgroups in childhood AML: a report from the French ELAM02 study group. HemaSphere. 2018;2: e31.

11. Kaplan E, Meier P. Nonparametric estimation from incomplete observations. J Am Stat Assoc. 1958;53:457-481.

12. Mantel N. Evaluation of survival data and two new rank order statistics arising in its consideration. Cancer Chemother Rep. 1966; 50:163-170.

13. Gouache E, Greze V, Leverger G. Clinical, biological, and prognostic characteristics of patients included in the ELAM02 study. HemaSphere. 2018;4.

14. Le Mouël L, Auvrignon A, Ragu C, et al. Outcome of infant acute myeloid leukemias in 3 successive trials: LAME 89/91, LAME 89/93 et ELAM02. Rev Oncol Hématologie Pédiatrique. 2015;3:175-187.

15. Creutzig U, Zimmermann M, Bourquin J-P, et al. Favorable outcome in infants with AML after intensive first- and second-line treatment: an AML-BFM study group report. Leukemia. 2012;26:654-661.

16. Webb DKH. Relationships between age at diagnosis, clinical features, and outcome of therapy in children treated in the Medical Research
Council AML 10 and 12 trials for acute myeloid leukemia. Blood. 2001;98:1714-1720.

17. Felix A, Leblanc $T$, Petit $A$, et al. Acute myeloid leukemia with central nervous system involvement in children: experience from the french protocol analysis ELAM02. J Pediatr Hematol Oncol. 2017;1.

18. Adinolfi M. The development of the human blood-CSF-brain barrier. Dev Med Child Neurol. 1985;27:532-537.

19. Pinkel D, Woo S. Prevention and treatment of meningeal leukemia in children. Blood. 1994;84:355-366.

20. Johnston DL, Alonzo TA, Gerbing RB, et al. Central nervous system disease in pediatric acute myeloid leukemia: a report from the Children's Oncology Group. Pediatr Blood Cancer. 2017;e26612.

21. Teyssier A-C, Lapillonne H, Pasquet M, et al. Acute megakaryoblastic leukemia (excluding Down syndrome) remains an acute myeloid subgroup with inferior outcome in the French ELAM02 trial. Pediatr Hematol Oncol. 2017;34:425-427.

22. Pui CH, Raimondi SC, Srivastava DK, et al. Prognostic factors in infants with acute myeloid leukemia. Leukemia. 2000;14:684-687.

23. Kawasaki $\mathrm{H}$. Superior outcome of infant acute myeloid leukemia with intensive chemotherapy: results of the Japan Infant Leukemia Study Group. Blood. 2001;98:3589-3594.

24. Masetti R, Rondelli R, Fagioli $F$, et al. Infants with acute myeloid leukemia treated according to the Associazione Italiana di Ematologia e Oncologia Pediatrica 2002/01 protocol have an outcome comparable to that of older children. Haematologica. 2014;99:e127-e129.

25. Balgobind BV, Hollink IHIM, Arentsen-Peters STCJM, et al. Integrative analysis of type-I and type-II aberrations underscores the genetic heterogeneity of pediatric acute myeloid leukemia. Haematologica. 2011;96:1478-1487.

26. Boissel N, Leroy $\mathrm{H}$, Brethon $\mathrm{B}$, et al. Incidence and prognostic impact of c-Kit, FLT3, and Ras gene mutations in core binding factor acute myeloid leukemia (CBF-AML). Leukemia. 2006;20:965-970.

27. Harrison CJ, Hills RK, Moorman AV, et al. Cytogenetics of childhood acute myeloid leukemia: united kingdom medical research council treatment trials AML 10 and 12. J Clin Oncol. 2010;28:2674-2681.

28. https://clinicaltrials.gov/ct2/show/NCT02724163. Principal Investigator: Brenda Gibson Royal Hospital for Children Glasgow First Posted: March 31, 2016 Last Update Posted: May 10, 2018. 